\title{
The impact of complications related to transvenous lead extraction on the 12-month prognosis: Insights from the SILCARD registry
}

\author{
Mateusz Tajstra ${ }^{1}$, Krzysztof Stanisław Golba ${ }^{2,3}$, Anna Kurek', Wojciech Jacheć, Ewa Nowolany-Kozielska ${ }^{4}$, \\ Michał Skrzypek ${ }^{5}$, Katarzyna Mizia-Stec ${ }^{6}$, Eugeniusz Piłat ${ }^{2}$, Anna Drzewiecka ${ }^{2}$, Artur Filipecki ${ }^{6}$, \\ Karol Przyłudzki' , Zbigniew Kalarus ${ }^{7}$, Mariusz Gąsior ${ }^{1}$, Adam Sokal ${ }^{7}$ \\ ${ }^{1} 3^{\text {rd }}$ Department of Cardiology, School of Medicine with the Division of Dentistry in Zabrze, Silesian Center for Heart Diseases, Medical University of Silesia, Katowice, \\ Poland \\ 2Department of Electrocardiology, Upper-Silesian Heart Center, Katowice, Poland \\ ${ }^{3}$ Department of Electrocardiology and Heart Failure, Medical University of Silesia, Katowice, Poland \\ ${ }^{4} 2^{\text {nd }}$ Department of Cardiology, Faculty of Medical Sciences in Zabrze, Medical University of Silesia, Katowice, Poland \\ ${ }^{5}$ Department of Biostatistics, School of Health Sciences in Bytom, Medical University of Silesia, Katowice, Poland \\ ${ }^{6} 1^{\text {st }}$ Department of Cardiology, Medical Faculty in Katowice, Medical University of Silesia, Katowice, Poland \\ ${ }^{7} 1^{\text {st }}$ Department of Cardiology and Angiology, Silesian Center of Heart Diseases Zabrze, Medical University of Silesia, Katowice, Poland
}

\author{
Correspondence to: \\ Mateusz Tajstra, MD, PhD, \\ $3^{\text {rd }}$ Department of Cardiology, \\ School of Medicine with \\ the Division of Dentistry in Zabrze, \\ Silesian Center for Heart Diseases, \\ Curie 9, 41-800 Zabrze, Poland, \\ phone: +48323733674, \\ e-mail: mateusztajstra@wp.pl \\ Copyright by the Author(s), 2022 \\ DOI: 10.33963/KP.a2021.0141 \\ Received: \\ July 14, 2021 \\ Accepted: \\ October 15, 2021 \\ Early publication date: \\ October 18, 2021
}

\section{A B S T R A C T}

Background: Scant data exist on long-term outcomes, including death, in patients with complications related to transvenous lead extractions (TLE).

Aims: We sought to characterize the population and examine the outcomes including risk factors for in-hospital complications and 12-month mortality and morbidity related to the complications in a large administrative database of patients undergoing TLE.

Results: From the database of patients hospitalized for cardiovascular diseases and included in the Silesian Cardiovascular Database (SILCARD) registry, we selected the admissions of those who underwent TLE according to the appropriate ICD-9 codes. The patients were divided into two groups based on whether they did or did not manifest any complications during their hospitalization for the TLE procedure. Between 2007 and 2019, we found a total of 835 patients who underwent TLE. TLE-related complications occurred in 56 patients $(6.7 \%)$ of the Complications-Yes group while no complications were recorded in 779 (93.3\%) patients of the Complications-No group. A significant difference in the rate of all-cause mortality $(23.9 \%$ vs. $6.5 \% ; P<0.001)$ and major adverse cardiac events (MACE) $(58.7 \%$ vs. $39.4 \% ; P=0.01)$ between the Complications-Yes and Complications-No groups were recorded. Multivariable analysis of the entire study population revealed that prior dialysis, chronic kidney disease, and ventricular tachycardia were independent factors of a higher risk of TLE-related in-hospital complications. Multivariable analysis of the patients discharged from the hospital after the TLE procedure showed that TLE-related complications, a history of heart failure, and older age independently affected 12-month mortality.

Conclusions: The presence of TLE-related in-hospital complications increased 12-month mortality.

Key words: complications, implantable electronic devices, lead extraction, registry, safety

\section{INTRODUCTION}

The number of procedures involving the use of cardiac implantable electronic devices (CIEDs) has increased significantly over recent years because of clear guidelines of interna- tional societies, improved access to healthcare, and a higher awareness of patients and their families [1, 2]. The natural implication of this increase is that the number of transvenous lead extractions (TLE) has also grown, as part 
WHAT'S NEW?

The presence of in-hospital complications related to transvenous lead extractions (TLE) independently increased 12-month mortality. The acquired data may allow clinicians to better stratify the risk of their patients, emphasize the importance of referral to experienced extraction centers, and indicate the need for closer clinical surveillance of patients after a complicated TLE procedure.

of an overall lead management strategy, in response to well-documented primarily infectious, but also non-infectious, indications and a considerable technical progression of the extraction equipment [3-5].

The largest-to-date prospective European Lead Extraction ConTRolled (ELECTRa) registry, containing the data of 3555 patients from 19 European countries, made it possible to draw many important conclusions in the TLE area [6], as stated in the expert consensus recently published by the European Heart Rhythm Association (EHRA). However, many issues remain unresolved and further clinical research and maintenance of national registries are needed [7].

An estimated 10000 to 15000 leads are extracted worldwide each year $[8,9]$. Notwithstanding the progress in new technologies and the increase in the safety of TLE owing to newer lead models, TLE is still considered a relatively high-risk and challenging procedure because of possible life-threatening complications [10]. Moreover, the data on the long-term comprehensive cardiovascular follow-up related to the impact of TLE complications on the prognosis and major adverse cardiac events (MACE) are scant.

Given the above, we sought to characterize the population and examine the outcomes, including risk factors for in-hospital complications and 12-month mortality and morbidity relative to the presence or absence of complications, using a large administrative database (Silesian Cardiovascular Database, SILCARD) of patients undergoing TLE. The identification of risk factors of in-hospital complications may help clinicians risk-stratify patients with indications for TLE.

\section{METHODS}

\section{Data source}

The purpose of the SILCARD registry (ClinicalTrials.gov identifier, NCT02743533) was described elsewhere [11]. In brief, the SILCARD registry was created under the agreement between the Silesian Center for Heart Diseases in Zabrze and the Silesian branch of the Polish National Health Fund (Narodowy Fundusz Zdrowia), the only health provider in Poland, to publish completed analyses of patients with cardiovascular diseases in the Province of Silesia (with a population of 3.7 million adults, 2 tertiary cardiology hospitals, 3 cardiac surgery departments, and 20 catheterization laboratories).

The SILCARD registry database contains data on all consecutive patients hospitalized in cardiology, cardiac sur- gery, vascular surgery, or diabetology units for any reason, or hospitalized in the internal medicine or intensive care units with the principal diagnosis of cardiovascular disease or with the diagnosis of a stroke at any neurology department in the Province of Silesia. Cardiovascular disease was defined as code R52 or J96 or any I code according to the International Statistical Classification of Diseases, Tenth Revision (ICD-10) [11].

\section{Study population and variables}

De-identified administrative hospital records were obtained from the Province of Silesia for the years 2007-2019. The data were harmonized and screened for admissions to identify subjects who underwent TLE, defined as having at least one of the following the International Classification of Diseases, $9^{\text {th }}$ Revision (ICD-9) codes in any of the 14 procedural code variables: 37.75 (revision of leads), 37.77 (removal of leads without replacement), or 37.97 (replacement of leads), at least one year after the implantation of the first device (pacemaker — ICD-9: 37.8, implantable cardioverter-defibrillator — ICD-9: 37.941-944; 37.961; $37.962 ; 37.991)$, and cardiac resynchronization therapy (ICD-9: 00.50; 00.51; 00.53; 0054), without any lead-related procedure during that period (Supplementary material, Table S1). The patients were divided into two groups based on any complications reported during the hospitalization for the TLE procedure: Complications-Yes, $n=56 ; 6.7 \%$ and Complications-No, $n=779 ; 93.3 \%$. Figure 1 represents the study flowchart. We excluded from the analysis patients under the age of 18 at the time of lead extraction. Based on the appropriate ICD-9 and ICD-10 codes (Supplementary material, Table S1), we analyzed baseline clinical characteristics of patients, the history of medical procedures, complications during TLE-related hospitalization, as well as MACE defined as a composite of all-cause death, stroke, or rehospitalization due to cardiovascular reasons (hospitalization with any principal diagnosis of cardiovascular disease) and procedures, reported in the 12-month follow-up after the TLE procedure for the entire cohort of patients and as a comparison between the Complications-Yes vs. the Complications-No groups. Twelve-month all-cause mortality was the primary endpoint of the study. All data were gathered anonymously so that individual cases could not be identified. The study complied with the Declaration of Helsinki. The locally appointed ethics committee approved the research protocol. The manuscript was reviewed and edited by all authors. All authors decided to submit the 


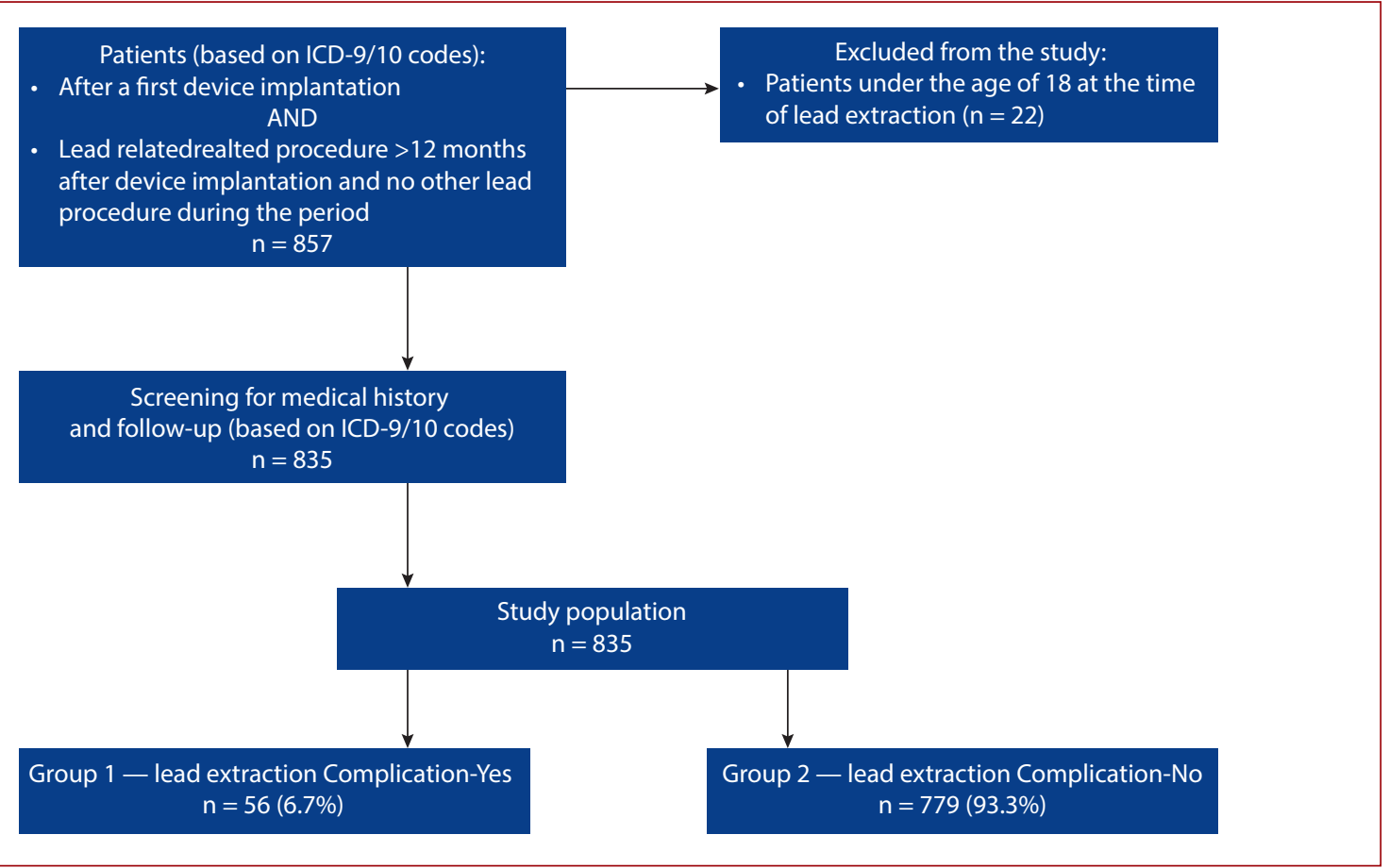

Figure 1. The study flow-chart. Step-by-step study population extraction

manuscript for publication and assume responsibility for the accuracy and completeness of the analyses.

\section{Statistical analysis}

Continuous variables are presented as means standard deviation (SD) or as medians with interquartile ranges (IQR). Categorical variables are presented as percentages. Continuous variables were compared using the T-test or the Mann-Whitney $U$ test where appropriate, whereas categorical variables were compared using a chi-squared test. The 12-month mortality and the MACE rate were analyzed using the Kaplan-Meier method with the log-rank test. A stepwise multiple logistic regression model was used to determine the predictors of TLE procedure-related complications and 12-month mortality after discharge, with the model including all the candidate variables except those with a high number of missing data. A significance level of $\leq 0.3$ was required to allow a variable into the model, and a significance level of $\leq 0.2$ was required for a variable to remain in the model. No interaction was tested. A two-sided $P$-value $<0.05$ was considered significant. The SAS software, version 9.4 (SAS Institute Inc., Cary, NC, USA) was used for all calculations.

\section{RESULTS}

Between January 2007 and December 2019, we identified a total of 835 patients who underwent lead extraction within more than 12 months after the first CIED implantation and were reported to the SILCARD registry (the mean number of 16.1 per million inhabitants, Figure 2). It should be emphasized that unambiguous indications for TLE were not reported.
Baseline demographic and clinical characteristics, type of lead, and department of the whole study group are presented in Supplementary material, Table S2. It should be emphasized that a typical spectrum of cardiovascular comorbidities and prior procedures/interventions for TLE patients were recorded. What is noteworthy is that more than $50 \%$ of lead extractions concerned patients after pacemaker implantation, and the mean age of extracted lead (SD) was 4.6 (2.9) years. In addition, nearly all TLEs were carried out in cardiology departments.

The 12-month outcomes of the entire study group are presented in Supplementary material, Table S3 - 65 deaths (7.8\%) were recorded while a MACE occurred in 334 (40\%) cases.

The data concerning complications during TLE hospitalization are listed in Table 1. The prevalence of all

Table 1. In-hospital complications

\begin{tabular}{l|c}
\multicolumn{1}{c|}{ Variable } & $\begin{array}{c}\text { Analyzed group } \\
(\mathbf{n = 8 3 5 )}\end{array}$ \\
All, $\mathrm{n}(\%)$ & $56(6.7)$ \\
Major, $\mathrm{n}(\%)$ & \\
All & $20(2.4)$ \\
Death & $10(1.2)$ \\
Stroke & $0(0)$ \\
Myocardial infarction & $3(0.4)$ \\
Cardiovascular lesions required pericardio- & $7(0.8)$ \\
centesis, emergent surgery & \\
Minor, $\mathrm{n}$ (\%) & \\
All & $49(5.8)$ \\
Pneumothorax required drainage & $6(0.7)$ \\
Hemothorax & $0(0)$ \\
Blood transfusion & $43(5.1)$ \\
\hline
\end{tabular}




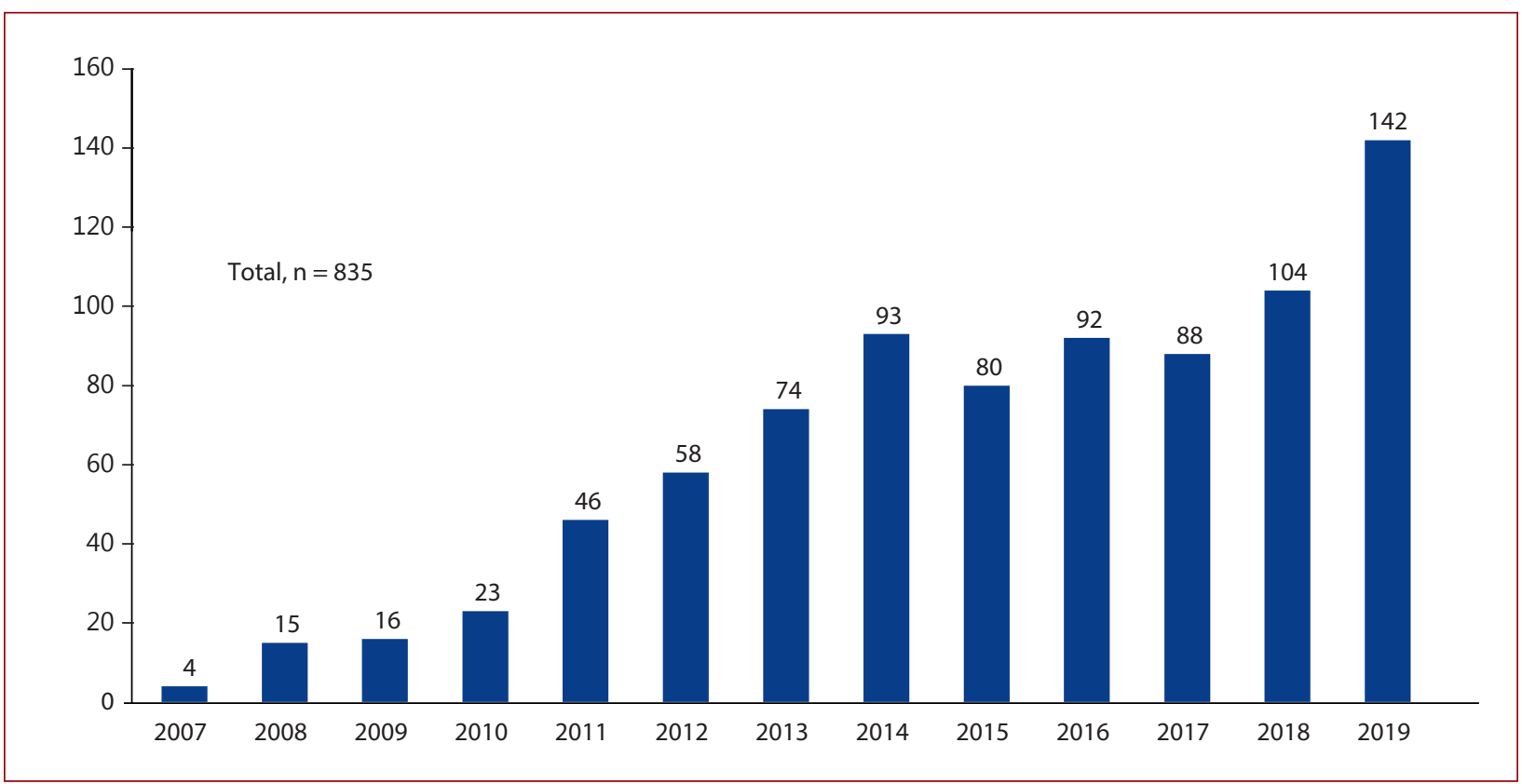

Figure 2. The number of patients with transvenous lead extraction in the Silesia region. Distribution of TLE procedures in particular years of the analysis

Abbreviations: TLE, transvenous lead extractions

Table 2. The baseline clinical characteristics, hospitalization stay length, and lead type of the study groups

\begin{tabular}{|c|c|c|c|}
\hline Variable & $\begin{array}{c}\text { Group } 1 \\
\text { Complications-Yes } \\
(n=56,6.7 \%)\end{array}$ & $\begin{array}{c}\text { Group } 2 \\
\text { Complications-No } \\
(n=779,93.3 \%)\end{array}$ & $P$-value \\
\hline Age, years, median (IQR) & $69.8(64.3-75.1)$ & $68.8(69.8-76.6)$ & 0.64 \\
\hline Male, n (\%) & $41(68.5)$ & $531(68.2)$ & 0.7 \\
\hline Dwelling time, years, median (IQR) & $3.8(2.1-6.5)$ & $4.0(2.1-6.5) 0.99$ & 0.99 \\
\hline Hospitalization length, days, median (IQR) & $30(17-48)$ & $6(4-10)$ & $<0.0001$ \\
\hline Lead type, n (\%) & & 0.37 & \\
\hline PM & $29(51.8)$ & $409(52.5)$ & \\
\hline ICD & $21(37.5)$ & $289(37.1)$ & \\
\hline CRT-D & $4(7.1)$ & $73(9.4)$ & \\
\hline CRT-P & $2(3.6)$ & $8(1)$ & \\
\hline \multicolumn{4}{|l|}{ Medical history, n (\%) } \\
\hline Heart failure & $44(78.6)$ & $515(66.1)$ & 0.05 \\
\hline Coronary artery disease & $42(75)$ & $514(76)$ & 0.2 \\
\hline Prior coronary angiography & $42(75)$ & $511(65.6)$ & 0.15 \\
\hline Prior myocardial infarction & $14(25)$ & $157(20.2)$ & 0.4 \\
\hline Prior percutaneous coronary intervention & $20(35.7)$ & $236(30.3)$ & 0.4 \\
\hline Prior coronary bypass & $3(5.4)$ & $46(5.9)$ & 1.0 \\
\hline Stroke & $5(8.9)$ & $46(5.9)$ & 0.4 \\
\hline Arterial hypertension & $43(76.8)$ & $496(63.7)$ & 0.05 \\
\hline Diabetes mellitus & $17(30.4)$ & $194(24.9)$ & 0.4 \\
\hline Chronic kidney disease & $11(19.6)$ & $29(3.7)$ & $<0.0001$ \\
\hline Prior dialysis & $7(12.5)$ & $4(0.5)$ & $<0.0001$ \\
\hline Prior infective endocarditis & $3(5.4)$ & $9(1.2)$ & 0.01 \\
\hline Atrial fibrillation/flutter & $29(51.8)$ & $304(39)$ & 0.06 \\
\hline Valvular heart disease & $28(50)$ & $331(42.5)$ & 0.2 \\
\hline Hypertrophic cardiomyopathy & $2(3.6)$ & $25(3.2)$ & 0.9 \\
\hline Sinus node dysfunction & $16(28.6)$ & $227(29.1)$ & 1.0 \\
\hline Atrio-ventricular block second or third degree & $11(19.6)$ & $185(23.7)$ & 0.5 \\
\hline Prior ventricular tachycardia & $16(28.6)$ & $139(17.8)$ & 0.05 \\
\hline Prior ventricular fibrillation & $2(3.6)$ & $40(5.1)$ & 0.1 \\
\hline Cardiac arrest & $3(5.4)$ & $43(5.5)$ & 0.9 \\
\hline Prior electrophysiology study & $3(5.4)$ & $25(3.2)$ & 0.4 \\
\hline Prior ablation & $5(8.9)$ & $47(6)$ & 0.4 \\
\hline
\end{tabular}

Abbreviations: CRT-D, cardiac resynchronization therapy-defibrillator; CRT-P, cardiac resynchronization therapy-peacemaker; ICD, implantable cardioverter-defibrillator; IQR, interquartile range; $\mathrm{PM}$, peacemaker 
Table 3. Predictors of the risk of in-hospital complications related to transvenous lead extraction (stepwise multiple logistic regression model results)

\begin{tabular}{l|c|c} 
& OR (95\% CI) & P-value \\
\hline Prior dialysis & $15.35(3.75-16.7)$ & $<0.001$ \\
Chronic kidney disease & $3.9(1.6-9.6)$ & 0.003 \\
Prior ventricular tachycardia & $2.15(1.14-4.05)$ & 0.02 \\
Prior infective endocarditis & $4.04(0.93-17.46)$ & 0.06 \\
Lead type PM vs. ICD/CRT-D & $3.19(0.82-12.32)$ & 0.09 \\
Heart failure & $1.98(0.85-3.92)$ & 0.12 \\
\hline
\end{tabular}

Abbreviations: $\mathrm{Cl}$, confidence interval; OR, odds ratio; other — see Table 2

major complications (death, stroke, myocardial infarction, emergent surgery) and minor complications (pocket revision, lead revision, a pneumothorax requiring drainage, a hemothorax, blood transfusion) was $2.4 \%(n=20)$ and $5.8 \%(n=49)$, respectively.

The baseline clinical characteristics, hospitalization length, and lead type in relation to the presence (Complications-Yes, $\mathrm{n}=56 ; 6.7 \%$ ) or absence (Complications-No, $n=779 ; 93.3 \%$ ) of post-TLE complications are presented in Table 2. Patients in the Complications-Yes group were more often affected by chronic kidney disease (19.6\% vs. 3.7\%; $P$ $<0.001)$, prior dialysis $(12.5 \%$ vs. $0.5 \%$; $P<0.001$,), and prior infective endocarditis ( $5.4 \%$ vs. $1.2 \% ; P=0.01$ ) than those in the Complications-No group. Additionally, there was a tendency towards a higher prevalence of heart failure $(78.6 \%$ vs. $66.1 \%$; $P=0.05)$, arterial hypertension (76.8\% vs. $63.7 \%$; $P=0.05)$, atrial fibrillation (51.8\% vs. $39 \% ; P=0.06)$, and prior ventricular tachycardia $(28.6 \%$ vs. $17.8 \%$; $P=0.05)$ in the Complications-Yes group. Hospitalization time was longer in the Complications-Yes group (median 30 days [IQR, 17-48] vs median 6 days [IQR, 4-10]; $P<0.001)$.

Multivariable analysis of the entire study population revealed that prior dialysis, chronic kidney disease, and ventricular tachycardia were independent factors of a higher risk of TLE-related in-hospital complications (Table 3).

The 12-month clinical outcomes in the analyzed groups are shown in Table 4. We observed a difference in the rate
Table 5. Predictors of the 12-month mortality in patients discharged after transvenous lead extraction procedure (stepwise multiple logistic regression model results)

\begin{tabular}{l|c|c} 
& OR $(\mathbf{9 5} \% \mathbf{C I})$ & $\boldsymbol{P}$-value \\
\hline TLE complications & $3.97(1.86-8.47)$ & $<0.001$ \\
Heart failure & $3.65(1.76-7.59)$ & $<0.001$ \\
Age (per one year more) & $1.04(1.015-1.65)$ & 0.001 \\
\hline
\end{tabular}

Abbreviations: TLE, transvenous lead extractions; other — see Table 3

of all-cause mortality $(23.9 \%$ vs. $6.5 \% ; P<0.001)$ and MACE (58.7\% vs. 39.4\%; $P=0.01$ ) between the Complications-Yes and Complications-No group (Figure 3; Supplementary material, Figure S1).

Multivariable analysis of patients discharged from the hospital after the TLE procedure showed that TLE-related complications, a history of heart failure, and older age independently affected 12-month mortality (Table 5).

\section{DISCUSSION}

Electrotherapy in the form of CIEDs plays an essential role in improving patients' prognosis and outcomes in bradyarrhythmias, conduction blocks, and HF. The number of implantations is likely to increase over time [2, 12]. Unfortunately, a serious challenge for electrotherapy is posed by mainly infectious, but also non-infectious, complications. Therefore, TLE, as a gold standard of treatment in many of them, has become an inseparable element of electrotherapy and the number of TLE procedures has been growing. Notwithstanding the technological progress in lead design and various TLE-dedicated tools, TLE remains a complex procedure, requiring high clinical attention. Consequently, an evaluation of TLE outcomes to understand the risk/safety ratio based on national registries may have important implications and provide a crucial source of indications regarding decision-making and therapeutic strategies in patients who are candidates for this procedure.

The principal clinical implications from the presented study can be summarized as follows. First, the number of TLE procedures has increased significantly each year with

Table 4. The 12-month outcomes for the study groups

\begin{tabular}{|c|c|c|c|}
\hline Variable, n (\%) & $\begin{array}{c}\text { Group } 1 \\
\text { Complications-Yes } \\
(n=56 ; 6.7 \%)\end{array}$ & $\begin{array}{c}\text { Group } 2 \\
\text { Complications-No } \\
\text { (n=779; } 93.3 \%)\end{array}$ & $P$-value \\
\hline \multicolumn{4}{|l|}{ Primary endpoint } \\
\hline Death $^{a}$ & $11(23.9)$ & $54(6.5)$ & $<0.001$ \\
\hline \multicolumn{4}{|l|}{ Secondary endpoints ${ }^{b}$} \\
\hline Stroke & $2(3.6)$ & $12(1.5)$ & 0.25 \\
\hline Re-hospitalization due to cardiovascular reason & $22(39.3)$ & $281(36.1)$ & 0.12 \\
\hline Major adverse cardiac events & $27(58.7)$ & $307(39.4)$ & $<0.01$ \\
\hline Pulmonary embolism & $0(0)$ & $3(0.4)$ & 1.0 \\
\hline Pocket revision & $3(5.4)$ & $17(2.2)$ & 0.13 \\
\hline Lead revision & $2(3.6)$ & $32(4.1)$ & 0.80 \\
\hline De-novo atrial fibrillation & $1(1.8)$ & $35(4.5)$ & 0.33 \\
\hline Infective endocarditis & $1(1.8)$ & $10(1.2)$ & 0.41 \\
\hline
\end{tabular}

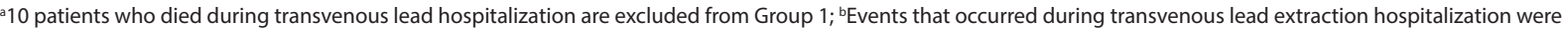
excluded from the analysis 


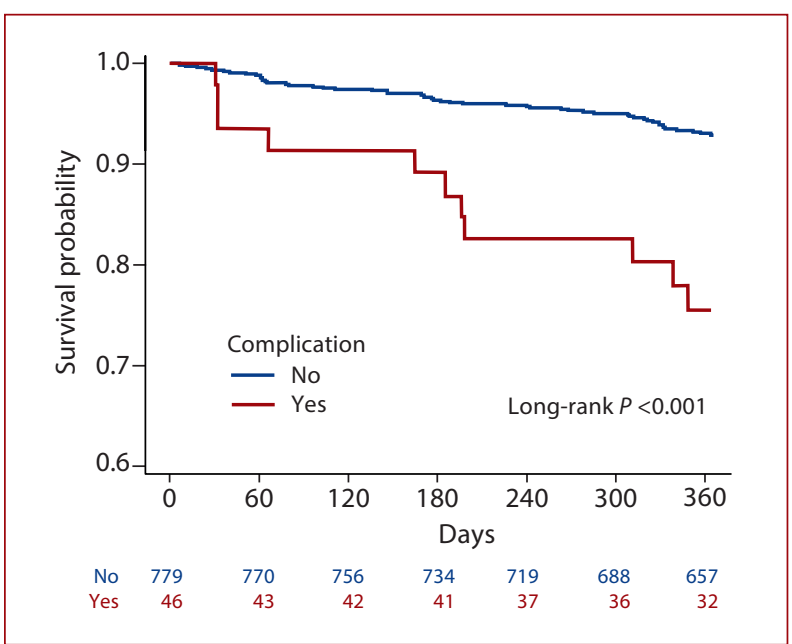

Figure 3. Twelve-month mortality of the study groups. The twelvemonth mortality rate was analyzed using the Kaplan-Meier method with the log-rank test

a mean rate of 16.1 per one million inhabitants in this highly urbanized area of Poland. Second, the rate of any complications and the rate of major complications during TLE-related hospitalization were low $(6.7 \%$ and $2.4 \%$, respectively). Third, the independent factors of TLE complications included prior dialysis, chronic kidney disease, and ventricular tachycardia. Finally, the occurrence of any TLE-related in-hospital complications increased the rate of 12-month mortality and MACE.

A total of 835 TLE procedures were performed during the study period in the Province of Silesia, in three cardiac surgery departments and 20 catheterization laboratories. The mean rate of 16.1 per one million is a bit higher than that included in the EHRA report (14.3 per one million procedures). However, the difference may be explained by missing or incomplete data, as stated by the authors of the EHRA report[1].

The baseline demographics including age (median age between 60 and 70 years), sex (about two-thirds were male), and medical history of this study population were comparable to other publications in the field of TLE [6, 13-15]. These similarities validate the population included in this study and allow further reference to prior reports.

The incidence of all major complications (2.4\%) including all-cause death (1.2\%) was low and in line with complication rates observed in previously published reports [13, $14,16]$. In the prospective ELECTRA registry by Bongiorni et al. [6], the rate of all-cause major complications and the rate of all-cause death were similar to our findings $(2.7 \%$ and $1.4 \%$, respectively). Although the prevalence of major complications was higher in some other studies (as high as $10 \%$ ), it seems that the difference is linked mostly to the design of other studies, which focused only on patients with TLE due to a device-related infection, a population that is no doubt at a higher risk of complications and death resulting from this procedure $[17,18]$.

Multiple studies have reported the presence of chronic kidney disease (CKD) and the previous dialysis to be independent predictors of complications after TLE [13, $19,20]$. Similarly, we observed more than 3.9-fold higher odds of in-hospital complications in patients with CKD and 15.3-times higher odds for those with prior dialysis.

Our findings show many similarities to other larger studies that have reported on risk factors for adverse events during TLE, but with some substantial exceptions. Although there is evidence that women may be more prone to adverse events following TLE, we found no relation between sex and procedural complications. It should be emphasized that this is similar to the findings published by Wazni et al.

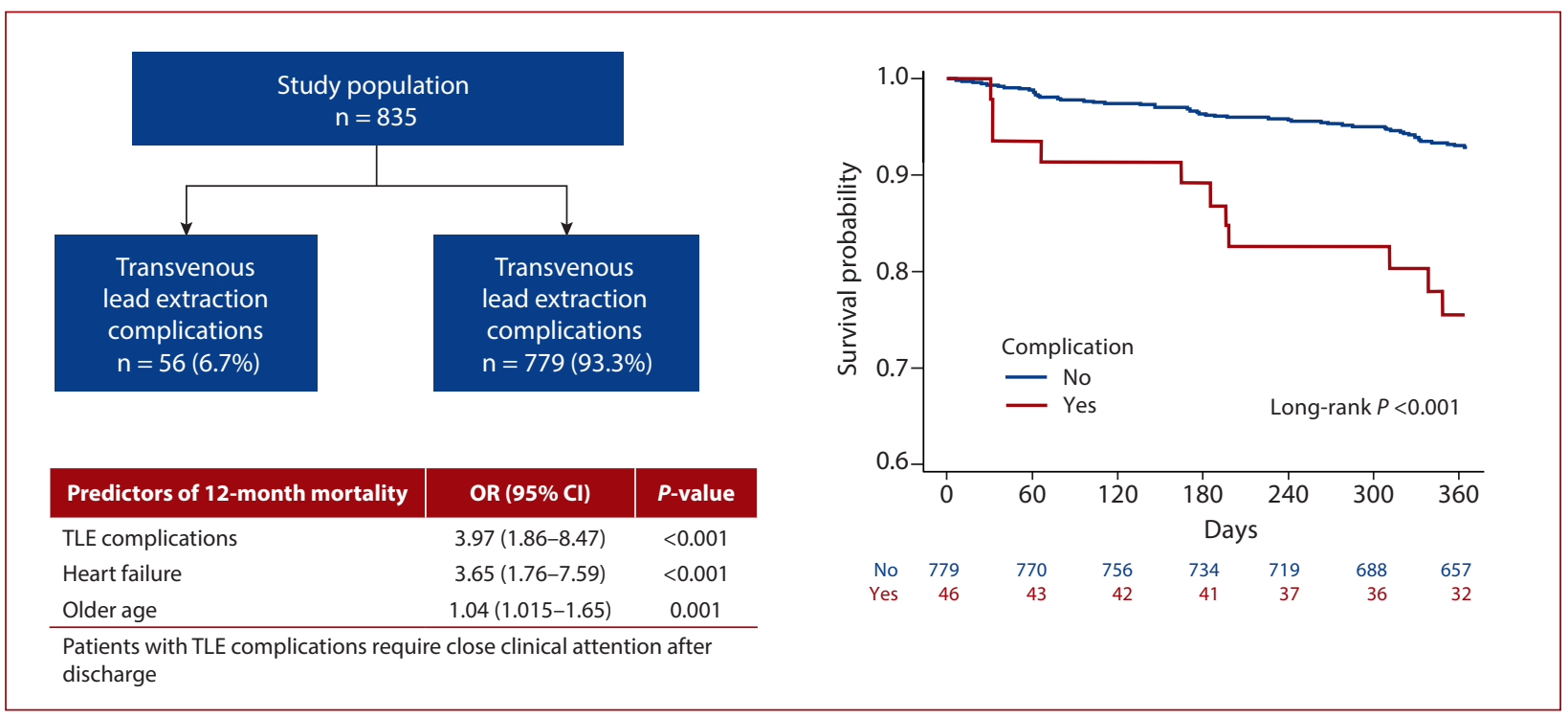

Figure 4. The most important findings of the study presented in a graphic form

Abbreviations: see Table 3 
[13]. Heart failure and lead age have previously been associated with an increased risk of TLE-related complications [6], although in our multivariable analysis they were not associated with adverse outcomes.

Besides previously identified well-known predictors, we discovered that TLE complications were more common in patients with a history of ventricular tachycardia. Although this is a novel finding, it is well recognized that patients with malignant ventricular arrhythmias are at a higher risk of major cardiovascular events [21]. Therefore, such patients may require more careful attention before being qualified for a complex TLE procedure, in the form of antiarrhythmic therapy escalation, electrolytes substitution, and coronary angiography or ablation procedures.

Several studies assessing long-term outcomes after TLE reported higher long-term mortality in infectious patients and other well-described independent factors affecting prognosis after the patient's discharge from the hospital [22-24]. Although these unambiguous indications for TLE were not reported in the presented study, to the best of our knowledge, none of the previously published reports focused directly on patients with complications during TLE hospitalization in terms of its impact on MACE including death in comparison to patients who underwent TLE without any reported significant events. Our analysis revealed that nearly four times more patients died within 12 months after discharge in the group with any TLE complications compared to patients who were complications-free. This fact confirms the pivotal role of TLE-procedure navigation, especially when some silent clinical complications occurred [25]. Additional studies are required to better understand the mechanisms by which procedural complications may adversely impact long-term outcomes.

\section{Limitations}

The study has several limitations. There is no unique ICD-9 code dedicated to the TLE procedure, which represents a major limitation of the study. The study is based on the electronic database of a single healthcare provider, and it is limited to core variables, such as demographic data, comorbidities, length of in-hospital stay, in-hospital and long-term morbidity and mortality. It does not cover data regarding laboratory results, echocardiographic parameters, pharmacotherapy, clear indications for TLE (infectious vs. non-infectious), type of infection, technical lead parameters, procedure details (operating theater, type of anesthesia, operator specialization, equipment, and methods for lead extraction used, number of extracted leads), the number of TLE procedures per center, and a center's TLE success rate. Although the main diagnosis according to the ICD-10 classification reported to the provider most often reflects the real reason for hospitalization, the reporting systems are not standardized, and the quality of data is challenged by the discrepancy between the details reported by different centers. Therefore, the data should be interpreted with caution.

\section{CONCLUSIONS}

The number of TLE procedures increased sharply from 2007 to 2013, reaching a plateau in the latter half of the analyzed period. Our study confirms the safety of the TLE procedures, which are associated with a low incidence of life-threatening complications. However, the occurrence of any TLE-related in-hospital complications increased 12-month mortality and the MACE rate. This latter finding suggests that patients with TLE complications discharged from hospitals require high clinical attention.

\section{Supplementary material}

Supplementary material is available at https://journals. viamedica.pl/kardiologia_polska.

\section{Article information}

Conflict of interest: None declared.

Open access: This article is available in open access under Creative Common Attribution-Non-Commercial-No Derivatives 4.0 International (CC BY-NC-ND 4.0) license, allowing to download articles and share them with others as long as they credit the authors and the publisher, but without permission to change them in any way or use them commercially. For commercial use, please contact the journal office at kardiologiapolska@ptkardio.pl.

\section{REFERENCES}

1. Raatikainen MJ, Arnar DO, Merkely B, et al. A decade of information on the use of cardiac implantable electronic devices and interventional electrophysiological procedures in the European Society of Cardiology countries: 2017 report from the European Heart Rhythm Association. Europace. 2017; 19(Suppl 2): ii1-ii90, doi: 10.1093/europace/eux258, indexed in Pubmed: 28903470.

2. Ponikowski P, Voors A, Anker S, et al. ESC Scientific Document Group. 2016 ESC Guidelines for the diagnosis and treatment of acute and chronic heart failure: The Task Force for the diagnosis and treatment of acute and chronic heart failure of the European Society of Cardiology (ESC) developed with the special contribution of the Heart Failure Association (HFA) of the ESC. Eur Heart J. 2016; 37(27): 2129-2200, doi: 10.1093/eurheartj/ehw128, indexed in Pubmed: 27206819.

3. Diemberger I, Mazzotti A, Giulia MB, et al. From lead management to implanted patient management: systematic review and meta-analysis of the last 15 years of experience in lead extraction. Expert Rev Med Devices. 2013; 10(4):551-573, doi: 10.1586/17434440.2013.811837, indexed in Pubmed: 23895081.

4. Bongiorni MG, Blomström-Lundqvist C, Kennergren C, et al. Scientific Initiative Committee, European Heart Rhythm Association. Current practice in transvenous lead extraction: a European Heart Rhythm Association EP Network Survey. Europace. 2012; 14(6): 783-786, doi: 10.1093/europace/eus166, indexed in Pubmed: 22622992.

5. Bongiorni MG, Marinskis G, Lip GYH, et al. Scientific Initiative Committee, European Heart Rhythm Association. How European centres diagnose, treat, and prevent CIED infections: results of an European Heart Rhythm Association survey. Europace. 2012; 14(11): 1666-1669, doi: 10.1093/europace/eus350, indexed in Pubmed: 23104858.

6. Bongiorni MG, Kennergren C, Butter C, et al. ELECTRa Investigators. The European Lead Extraction ConTRolled (ELECTRa) study: a European Heart Rhythm Association (EHRA) Registry of Transvenous Lead Extraction Outcomes. Eur Heart J. 2017; 38(40): 2995-3005, doi: 10.1093/eurheartj/ehx080, indexed in Pubmed: 28369414.

7. Bongiorni MG, Burri H, Deharo JC, et al. ESC Scientific Document Group. 2018EHRA expert consensus statement on lead extraction: recommendations on definitions, endpoints, research trial design, and data collection requirements for clinical scientific studies and registries: endorsed by APHRS/HRS/LAHRS. Europace. 2018; 20(7): 1217, doi: 10.1093/europace/euy050, indexed in Pubmed: 29566158. 
8. Hauser RG, Katsiyiannis WT, Gornick CC, et al. Deaths and cardiovascular injuries due to device-assisted implantable cardioverter-defibrillator and pacemaker lead extraction. Europace. 2010; 12(3): 395-401, doi: 10.1093/europace/eup375, indexed in Pubmed: 19946113.

9. Maytin $M$, Epstein LM, Henrikson CA. Lead extraction is preferred for lead revisions and system upgrades: when less is more. Circ Arrhythm Electrophysiol. 2010; 3(4): 413-424, doi: 10.1161/CIRCEP.110.954107, indexed in Pubmed: 20716723.

10. Jacheć W, Polewczyk A, Polewczyk M, et al. Transvenous lead extraction SAFETY score for risk stratification and proper patient selection for removal procedures using mechanical tools. J Clin Med. 2020; 9(2): 361, doi: 10.3390/jcm9020361, indexed in Pubmed: 32013032.

11. Gąsior M, Pres D, Wojakowski W, et al. Causes of hospitalization and prognosis in patients with cardiovascular diseases. Secular trends in the years 2006-2014 according to the SILesian CARDiovascular (SILCARD) database. Pol Arch Med Wewn. 2016; 126(10): 754-762, doi: 10.20452/pamw.3557, indexed in Pubmed: 27650214.

12. Brignole M, Auricchio A, Baron-Esquivias G, et al. ESC Committee for Practice Guidelines (CPG), Document Reviewers. 2013 ESC Guidelines on cardiac pacing and cardiac resynchronization therapy: the Task Force on cardiac pacing and resynchronization therapy of the European Society of Cardiology (ESC). Developed in collaboration with the European Heart Rhythm Association (EHRA). Eur Heart J. 2013; 34(29): 2281-2329, doi: 10.1093/eurheartj/eht150, indexed in Pubmed: 23801822.

13. Wazni O, Epstein LM, Carrillo RG, et al. Lead extraction in the contemporary setting: the LExICon study: an observational retrospective study of consecutive laser lead extractions. J Am Coll Cardiol. 2010; 55(6):579-586, doi: 10.1016/j.jacc.2009.08.070, indexed in Pubmed: 20152562.

14. Sood N, Martin DT, Lampert R, et al. Incidence and predictors of perioperative complications with transvenous lead extractions: real-world experience with national cardiovascular data registry. Circ Arrhythm Electrophysiol. 2018; 11(2): e004768, doi: 10.1161/CIRCEP.116.004768, indexed in Pubmed: 29453324.

15. Hosseini SM, Rozen G, Kaadan MI, et al. Safety and in-hospital outcomes of transvenous lead extraction for cardiac implantable device-related infections: analysis of 13 years of inpatient data in the United States. JACC Clin Electrophysiol. 2019; 5(12): 1450-1458, doi: 10.1016/j.jacep.2019.08.020, indexed in Pubmed: 31857046.

16. Katarzyńska-Szymańska A, Grymuza M, Chmielewska-Michalak L, et al. Implementation of laser equipment in a center experienced in lead extraction: safety and efficacy within 1-year follow-up. Kardiol Pol. 2021; 79(5): 569-571, doi: 10.33963/KP.15983, indexed in Pubmed: 34125932.

17. Merchant FM, Levy MR, Kelli HM, et al. Predictors of long-term survival following transvenous extraction of defibrillator leads. Pacing Clin Electrophysiol. 2015; 38(11): 1297-1303, doi: 10.1111/pace.12733, indexed in Pubmed: 26256667.

18. Brunner MP, Yu C, Hussein AA, et al. Nomogram for predicting 30-day all-cause mortality after transvenous pacemaker and defibrillator lead extraction. Heart Rhythm. 2015; 12(12): 2381-2386, doi: 10.1016/j. hrthm.2015.07.024, indexed in Pubmed: 26190318.

19. Barakat AF, Wazni OM, Tarakji KG, et al. Transvenous lead extraction in chronic kidney disease and dialysis patients with infected cardiac devices. Circ Arrhythm Electrophysiol. 2018; 11(1): e005706, doi: 10.1161/CIRCEP.117.005706, indexed in Pubmed: 29321192.

20. Lekkerkerker JC, van Nieuwkoop C, Trines SA, et al. Risk factors and time delay associated with cardiac device infections: Leiden device registry. Heart. 2009; 95(9): 715-720, doi: 10.1136/hrt.2008.151985, indexed in Pubmed: 19036758.

21. Gomes JA, Mehta D, Ip J, et al. Predictors of long-term survival in patients with malignant ventricular arrhythmias. Am J Cardiol. 1997; 79(8): 1054-1060, doi: 10.1016/s0002-9149(97)00046-5, indexed in Pubmed: 9114763.

22. Aleong RG, Zipse MM, Tompkins $C$, et al. Analysis of outcomes in 8304 patients undergoing lead extraction for infection. J Am Heart Assoc. 2020; 9(7): e011473, doi: 10.1161/JAHA.118.011473, indexed in Pubmed: 32192410.

23. Tarakji KG, Wazni OM, Harb S, et al. Risk factors for 1-year mortality among patients with cardiac implantable electronic device infection undergoing transvenous lead extraction: the impact of the infection type and the presence of vegetation on survival. Europace. 2014; 16(10): 1490-1495, doi: 10.1093/europace/euu147, indexed in Pubmed: 25087154.

24. Maytin $\mathrm{M}$, Jones SO, Epstein LM. Long-term mortality after transvenous lead extraction. Circ Arrhythm Electrophysiol. 2012; 5(2): 252-257, doi: 10.1161/CIRCEP.111.965277, indexed in Pubmed: 22362891.

25. Nowosielecka D, Polewczyk A, Jacheć W, et al. Transesophageal echocardiography for the monitoring of transvenous lead extraction. Kardiol Pol. 2020; 78(12): 1206-1214, doi: 10.33963/kp.15651, indexed in Pubmed: 33078921. 